\title{
The Development of Deformation Textures Described by an Orientation Flow Field
}

\author{
H. J. BUNGE†, C. ESLING $\ddagger$, E. DAHLEM $\dagger$ and H. KLEIN $\dagger$ \\ $\dagger$ Institut für Metallkunde und Metallphysik der TU Clausthal, F.R.G. \\ \$Laboratoire de Métallurgie Structurale, Université de Metz, France.
}

(Received November 30, 1985)

The mean orientation change $\delta g$ of crystals of the orientation $g$ in a polycrystalline material due to plastic deformation can be described (under certain assumptions) by an orientation flow field $\delta g(g)=d \eta \cdot v(g)$ where $d \eta$ is the absolute value of a small deformation step. The flow field $v(g)$ is a vector field in the orientation space $g=\left\{\varphi_{1}, \Phi, \varphi_{2}\right\}$ which must obey a continuity equation. The flow field describes the texture changes due to plastic deformation. The components of the flow vector, as a function of the orientation $g$, can be represented in terms of a series expansion which must obey certain symmetry conditions. As an example, the flow field calculated according to the Taylor theory for $\{111\}\langle 110\rangle$ glide was calculated in 5-degree steps in the orientation space.

\section{INTRODUCTION}

If a polycrystalline material is being deformed plastically this generally leads to the development of a deformation texture or, more generally speaking, to the modification of the existing texture of the material. This is mainly due to two processes, i.e. glide and twinning, by which crystal orientation is being changed either continuously or discontinuously. In the present paper we shall consider the orientation changes and hence texture changes by glide processes only. Since the glide systems are crystallographically oriented they have different orientations with respect to the macroscopic strain axes in each grain. If one takes also the strain continuity and stress equilibrium conditions in the 
grain boundaries into account then this leads to an inhomogenous distribution of the activated glide systems, to inhomogeneous strains and to inhomogeneous orientation changes not only from grain to grain but also within each grain. If, however, one does not consider the individual grain but only the statistical mean value of the behaviour of a great number of grains then a mean orientation change of all grains having the same orientation can be defined. It is represented by an orientation flow field in the orientation space.

\section{Definition of the flow field}

During plastic deformation a point $\vec{x}$ of the material is shifted into $\vec{x}^{\prime}$, Fig. 1, such that

$$
\vec{x}-\vec{x}=\vec{u}\left(x_{1}, x_{2}, x_{3}\right) \quad \vec{u}=\left\{u_{1}, u_{2}, u_{3}\right\}
$$

where $\vec{u}$ is a displacement vector. The local deformation of the material in the vicinity of the point $\vec{x}$ is described by the displacement gradient tensor

$$
\frac{\partial u_{i}}{\partial x_{j}}=e_{i j}\left(x_{1}, x_{2}, x_{3}\right)
$$

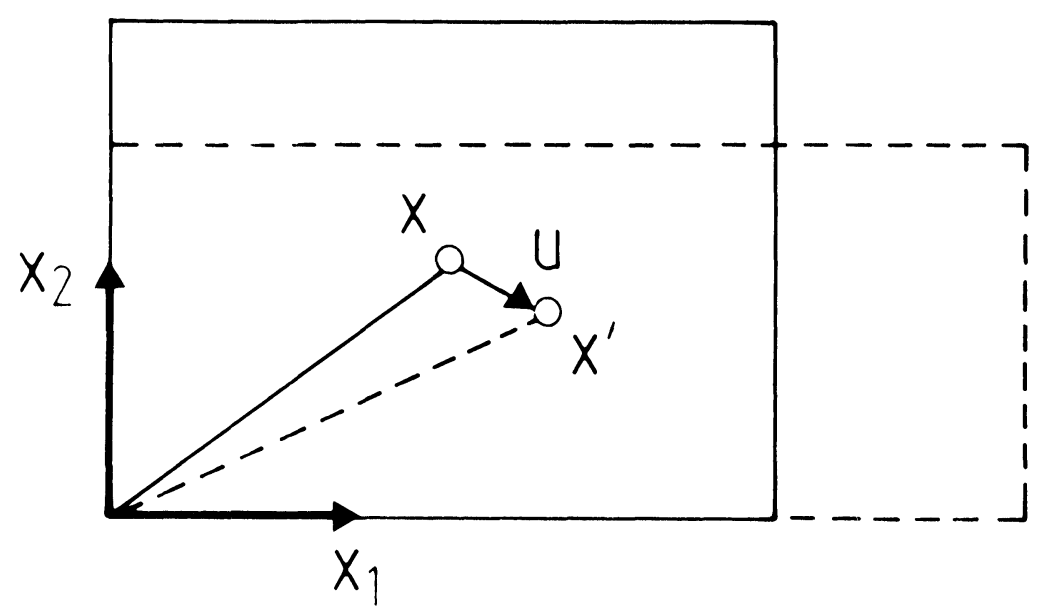

FIGURE 1 During plastic deformation a point $\vec{X}$ of the material is displaced by the vector $\vec{u}$ into $\vec{X}^{\prime}$. 
The deformation is generally composed of an elastic and a plastic part

$$
e_{i j}=e_{i j}^{e}+e_{i j}^{p}
$$

If we consider texture changes by plastic deformation then the elastic part is usually small compared with the plastic part. (see e.g. Kocks 1970) Hence, we may assume in the following

$$
e_{i j} \approx e_{i j}^{p}
$$

We consider a macroscopically homogenous deformation characterized by the mean value of the local displacement gradient

$$
\bar{e}_{i j}=\frac{1}{V} \int e_{i j}\left(x_{1}, x_{2}, x_{3}\right) d x_{1} d x_{2} d x_{3}
$$

which is enforced upon the material by the deformation tool. We further consider a small deformation step. Then $e_{i j}$ may be split into an absolute value $d \eta$ of the deformation and a tensor $E_{i j}$ discribing the deformation geometry

$$
d e_{i j}=d \eta \cdot E_{i j}
$$

Eq. 6 may be applied to the local displacement gradient $e_{i j}\left(x_{1}, x_{2}, x_{3}\right)$ as well as to its mean value $\bar{e}_{i j}$. If $E_{i j}$ is the same in all consecutive deformation steps then eq. 6 can be integrated

$$
e_{i j}=\eta \cdot E_{i j}
$$

where

$$
\eta=\int d \eta
$$

is the degree of deformation e.g. the rolling degree.

The crystallographic orientation of the material in the point $\vec{x}$ may be described by the rotation $g$ of a crystal coordinate system with respect to a sample coordinate system, Fig. 2

$$
g=g\left(x_{1}, x_{2}, x_{3}\right) \quad g=\left\{\varphi_{1}, \Phi, \varphi_{2}\right\}
$$

The texture of the material is then defined by the volume fraction of the material having an orientation $g$ within the limits $d g$ (see e.g. Bunge 1982)

$$
\frac{d V(g)}{V}=f(g) d g
$$




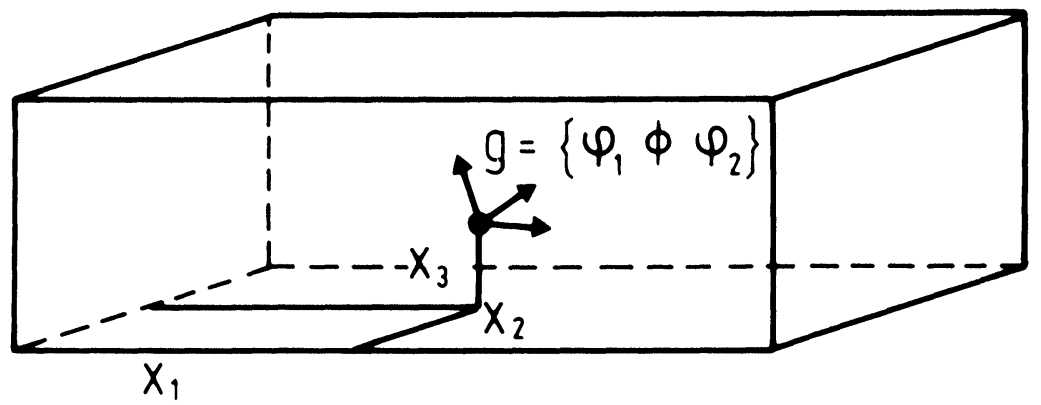

FIGURE 2 The orientation $g=\left\{\varphi_{1}, \Phi, \varphi_{2}\right\}$ in the point $\vec{X}=\left\{x_{1}, x_{2}, x_{3}\right\}$ of the material.

As a consequence of the deformation the orientation $g$ in the point $\vec{x}$ changes into $g^{\prime}$ in the point $\vec{x}^{\prime}$ such that

$$
g^{\prime}\left(x_{1}{ }^{\prime}, x_{2}{ }^{\prime}, x_{3}{ }^{\prime}\right)-g\left(x_{1}, x_{2}, x_{3}\right)=\Delta g\left(x_{1}, x_{2}, x_{3}\right)
$$

The orientation change $\Delta g$ depends on the local deformation tensor $e_{i j}$, on the local orientation $g$, on the actual slip systems $\{h k l\}\langle u v w\rangle$ by which deformation takes place and this in turn may depend on the orientation correlation between the point $\vec{x}$ and its environment. With the macroscopic deformation $\bar{e}_{i j}$ and the crystallographic type of the slip systems $\{h k l\}\langle u v w\rangle$ fixed, the orientation change $\Delta g$ is thus not a unique function of the local orientation $g$ (see e.g. Gil Sevillano et al. 1980).

In order to consider texture changes by plastic deformation we consider the mean value of $\Delta g$ over all volume elements having the same orientation $g$

$$
\delta g=\frac{1}{d V(g)} \int_{g} \Delta g(\vec{x}) d x_{1} d x_{2} d x_{3}=\left\{\delta \varphi_{1}, \delta \Phi, \delta \varphi_{2}\right\}
$$

where $d V(g)$ is the volume defined in eq. 10 .

We consider a small deformation step $d \eta$ as in eq. 6 then an orientation flow field can be defined by

$$
\delta g=d \eta \cdot v(g), v=\left\{v_{1}, v_{2}, v_{3}\right\}
$$

The flow field depends on the macroscopic deformation $\bar{E}_{i j}$, on the crystallographic type of the glide systems $\{h k l\}\langle u v w\rangle$ and their respective critical resolved shear stresses $\tau_{0}^{i}$ (including hardening and possible latend hardening) and in the most general case it may also depend explicitly on the texture $f(g)$. The latter possibility will, however, 
not be considered in the following. Under these assumptions a flow field as a function of the orientation $g$ may be defined which depends on the macroscopic strain tensor $\bar{E}_{i j}$, the nature of the glide systems $\{h k l\}\langle u v w\rangle$ and their respective critical shear stresses $\tau_{0}^{i}$ which in turn may be a function of the degree of deformation.

$$
v=v(g)_{E_{i},\{h k l\}\langle u v w\rangle, \tau_{0}^{i}(\eta)}
$$

The flow field as a function of $g$ is thus represented by a vector field in the orientation space, Fig. 3 (Bunge, Esling, 1984).

The flow field may have zero points. These correspond to orientations $g_{0}$ which are deformed without orientation changes. If, in the vicinity of $g_{0}$, the flow field $v(g)$ is oriented towards $g_{0}$ then $g_{0}$ is considered to

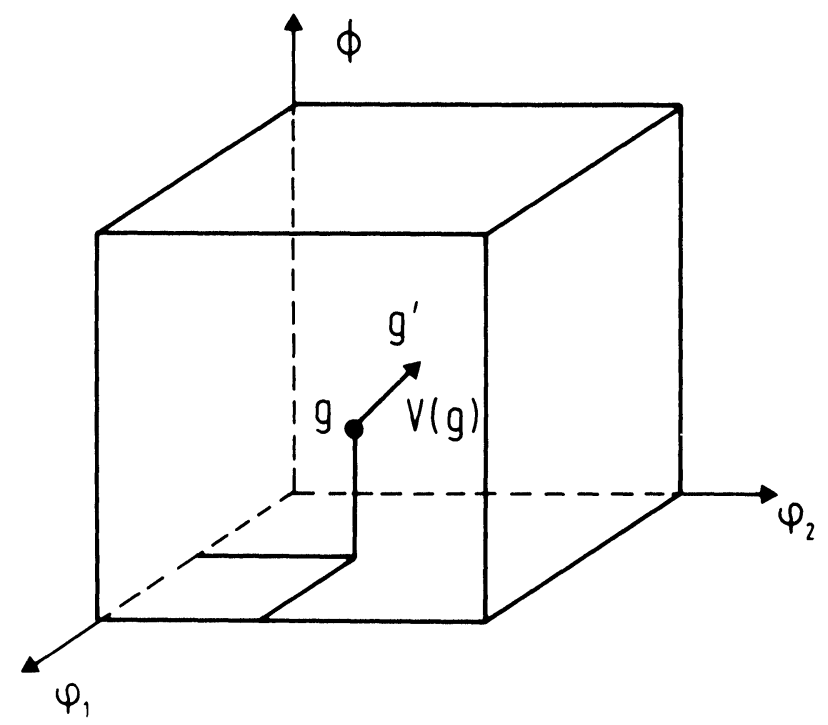

FIGURE 3 During plastic deformation the crystal orientation $g$ is shifted by $v(g)$ into $g^{\prime}$.

be a stable end orientation of the deformation process. If $v(g)$ is oriented away from $g_{0}$ then $g_{0}$ is an unstable zero orientation and finally also metastable orientations may occur which are partly stable and partly unstable as is shown schematically in Fig. 4.

The flow field leads to a texture change. Since the total orientation density (integrated over the whole orientation space) must be conserved and since we assumed only continuous orientation changes (described by a continuous flow field) the general continuity equation must be 


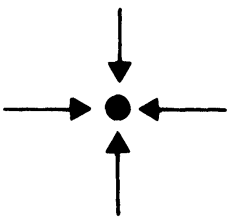

stable

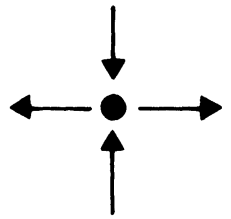

metastable

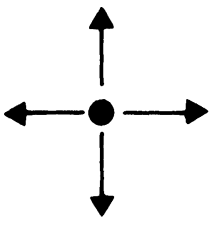

unstable

FIGURE 4 The flow field $v(g)$ may contain stable, metastable and unstable zero points.

valid which can be written in terms of the texture and the flow field (Clement and Coulomb (1978), (1979), Clement (1980))

$$
\operatorname{div}\{f(g, \eta) \cdot v(g, \eta)\}=-\frac{\partial f(g, \eta)}{\partial \eta}
$$

where it has been admitted that the flow field itself may change during the deformation process for example by a change of the critical resolved shear stresses of the glide system due to anisotropic hardening.

The flow field may be measured experimentally by deforming single crystals in various orientations $g$ with a deformation tensor according to eq. 5. A great deal of measurements of this kind have been carried out but not in a way covering systematically the whole orientation space $g$ in sufficiently small steps as would be necessary for the construction of a complete orientation flow field.

The flow field may also be calculated using a theoretical model of plastic deformation. Several models of plastic deformation have been considered among which the Taylor theory and its recent generalizations seem to be most successful. Surveys on the various models have been given by Kocks (1970), Gil Sevillano et al. (1980), Berveiller and Zaoui (1978), Mecking (1981), van Houtte (1984).

\section{Calculation of the flow field according to the Taylor theory}

The flow field $v(g)$ has been calculated with the assumptions of the Taylor theory (Bunge 1970). In this theory it is assumed that the 
deformation of the polycrystalline material is homogeneous i.e. the local plastic deformation is equal to the macroscopic one (Taylor 1938).

$$
\mathrm{de}_{i j}\left(x_{1}, x_{2}, x_{3}\right)=\mathrm{de}_{i j}=d \eta \bar{E}_{i j}
$$

Furthermore, the deformation is composed of homogeneous shear deformations corresponding to glide in the glide systems and a possible lattice rotation

$$
\bar{E}_{i j}=\sum_{n=1}^{N} \gamma^{n} E_{i j}^{n}(g)+R_{i j}(g)
$$

where $\gamma^{n}$ is the amount of glide in the n-th glide system, $E_{i j}^{n}$ is the deformation tensor of unit glide in this system and $R_{i j}$ is an antisymmetric tensor describing the lattice rotation which is directly related to the flow field $v(g)$.

In a coordinate system, the $X_{1}^{\prime \prime}$-axis of which is the glide direction and the $X_{3}^{\prime \prime}$-axis being the normal to the glide plane the glide tensor $E^{n}$ has the components

$$
E_{i j}^{\prime \prime}=\left[\begin{array}{lll}
0 & 0 & 1 \\
0 & 0 & 0 \\
0 & 0 & 0
\end{array}\right]
$$

If the glide direction of the $\mathrm{n}$-th glide system is described by a unit vector $\vec{d}^{n}$ with the components $d_{i}^{n}$ in the crystal coordinate system and a unit vector $\overrightarrow{p^{n}}$ normal to the glide plane which has the components $p_{j}^{n}$ then the glide tensor has the components

$$
E_{i j}^{\prime n}=d_{i}^{n} \cdot p_{j}^{n}
$$

in the crystal coordinate system. And finally the components with respect to the sample coordinate system are

$$
E_{i j}^{n}(g)=a_{i k}(g) \cdot a_{j i}(g) \cdot d_{k}^{n p n}
$$

where $a_{i k}(g)$ are the components of the transformation matrix transforming the sample coordinate system into the crystal coordinate system. They can be expressed by the Euler angles, given in eq. 9 .

$a_{i k}\left(\varphi_{1} \Phi \varphi_{2}\right)=\left[\begin{array}{l|l|l}\cos \varphi_{1} \cos \varphi_{2} & \sin \varphi_{1} \cos \varphi_{2} & \sin \varphi_{2} \sin \Phi \\ -\sin \varphi_{1} \sin \varphi_{2} \cos \Phi & +\cos \varphi_{1} \sin \varphi_{2} \cos \Phi & \\ \hline-\cos \varphi_{1} \sin \varphi_{2} & -\sin \varphi_{1} \sin \varphi_{2} & \cos \varphi_{2} \sin \Phi \\ -\sin \varphi_{1} \cos \varphi_{2} \cos \Phi & +\cos \varphi_{1} \cos \varphi_{2} \cos \Phi & \\ \hline \sin \varphi_{1} \sin \Phi & -\cos \varphi_{1} \sin \Phi & \cos \Phi\end{array}\right]$ 
Eq. 17 can be split into a symmetric and an antisymmetric part

$$
\begin{gathered}
\bar{E}_{i j}^{s}=\sum_{n=1}^{N} \gamma^{n} E_{i j}^{n s}(g) \\
\bar{E}_{i j}^{a}=\sum_{n=1}^{N} \gamma^{n} E_{i j}^{n \alpha}(g)+R_{i j}(g)
\end{gathered}
$$

where

$$
\bar{E}_{i j}^{s}=\bar{E}_{j i}^{s} ; \quad \bar{E}_{i j}^{a}=-E_{j i}^{a}
$$

Because of eq. 22, the symmetric tensor $\bar{E}_{i j}^{s}$ has six independent components and since plastic deformation does not change the volume it is

$$
\sum_{i=1}^{3} \bar{E}_{i i}^{s}=0
$$

Hence, eq. 17a consists of five independent linear equations with $N$ unknows $\gamma^{n}$, the amounts of glide in the glide systems.

If we consider for example the $\{111\}\langle 110\rangle$ glide systems in fcc metals then it is $N=12$. The linear system eq. $17 \mathrm{a}$ is thus strongly underdetermined. According to the Taylor theory eq. 17a is then to be solved under the assumption of minimum deformation work (Taylor 1938).

$$
W=\sum_{n=1}^{N} \tau_{0}^{n}\left|\gamma^{n}\right|=\operatorname{Min}
$$

The amounts of glide $\gamma^{n}$ in the $N$ glide systems are obtained by solving eq. $17 \mathrm{a}$ under the condition eq. 24.

According to the original Taylor theory a solution to eq. 17a can be obtained by allowing only 5 of the $\gamma^{n}$ to be different from zero which may be denoted by $\gamma^{n i}$. Out of the so obtained $\left(\begin{array}{c}N \\ 5\end{array}\right)$ solutions of eq. $17 \mathrm{a}$, the ones that minimize eq. 24 have then to be selected. In the general case there will be $M$ solutions $\gamma_{m}^{n}$ of eq. 17a that minimize eq. 24 each of which contains, at the most, 5 non-zero values.

The rotation matrices $R_{i j}(g)$ are then obtained from eq. $17 \mathrm{~b}$ with the $\gamma_{m}^{n}$ according to eq. $17 \mathrm{a}$ and 24 .

$$
R_{i j}^{m}(g)=\bar{E}_{i j}^{a}-\sum_{n=1}^{N} \gamma_{m}^{n} E_{i j}^{n a}(g)
$$

The lattice rotation is thus, in general, not unique for a given orientation $g$. Rather, any rotation 


$$
R_{i j}(g)=\sum_{m=1}^{M} \alpha_{m} R_{i j}^{m}(g)
$$

is possible where $\alpha_{m}$ are positive coefficients such that

$$
\sum_{m=1}^{M} \alpha_{m}=1
$$

The flow field in the orientation space is thus not unique as is shown schematically in Fig. 5.

If one considers texture formation by plastic deformation then it may

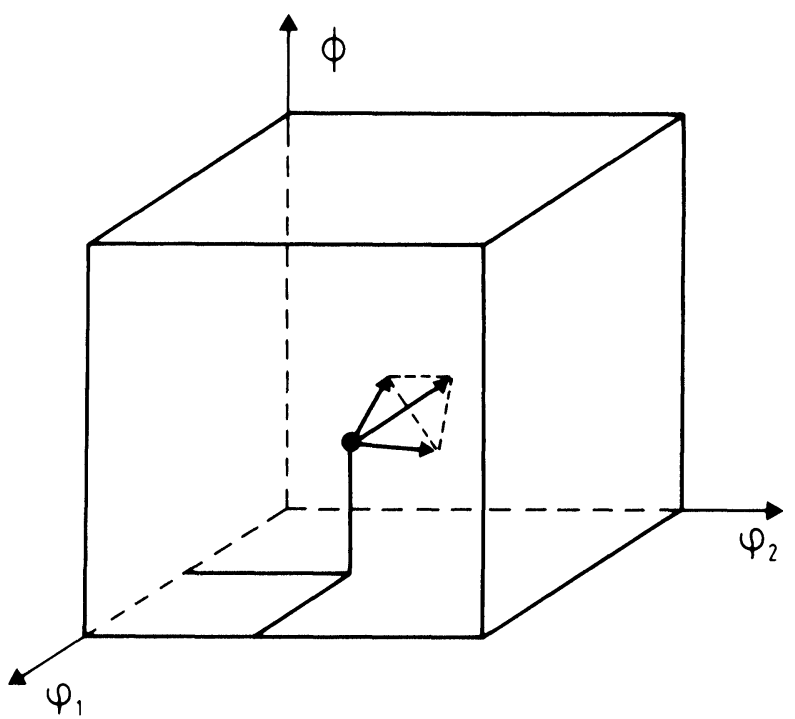

FIGURE 5 According to the Taylor theory a crystal orientation $g$ may be changed in different ways according to the solutions $R_{i j}^{m}(g)$ eq. 25 .

be assumed that the degeneration of the solution to eq. $17 \mathrm{a}, 24$, expressing itself in the arbitrary coefficients $\alpha_{m}$, is removed by the influence of the environment of the considered volume element such that each volume element assumes a specific solution out of eq. 26 . The assumption may then be made that the individual solutions $\gamma_{m}^{n}$ and hence $R_{i j}^{m}$ are equally frequent. Hence, a mean lattice rotation is obtained (Bunge 1970) by

$$
\bar{R}_{i j}(g)=\frac{1}{M} \sum_{m=1}^{M} R_{i j}^{m}(g)
$$


which corresponds to a unique flow vector $v(g)$ in the orientation space as was shown in Fig. 3. Also other assumptions have been made, leading to other coefficients $\alpha_{m}$ (see e.g. Chin 1969).

An antisymmetric tensor represents a rigid rotation about a rotation axis which may be represented by a rotation vector $\vec{R}$ with the components

$$
R_{1}^{s}=R_{23} ; R_{2}^{s}=R_{31} ; R_{3}^{s}=R_{12}
$$

the absolute value of which is related to the rotation angle $\omega$ in the case of infinitesimal rotations as is assumed here by

$$
|R|=2 \sin \frac{\omega}{2} \sim \omega
$$

The antisymmetric tensor $R_{i j}$ in eq. 17 was expressed in components with respect to the sample coordinate systems. Hence, also $R_{i}^{s}$ in eq. 29 are components of the rotation vector in the sample coordinate system which is indicated by the index $s$. The rotation vector may also be expressed in the crystal coordinate system. It is then

$$
R_{i}^{c}=a_{j i} \cdot R_{j}^{s}
$$

where $a_{j i}$ is the transposed of the transformation matrix eq. 21 . The rotation tensor $R_{i j}(g)$ eq. 17 is defined for a deformation of the absolute value $d \eta=1$. Hence, it corresponds to the orientation change $v(g)$ defined in eq. 13. For a small value of $d \eta$ the components of $v(g)$ in the space of the Euler angles can be expressed by (Bunge 1970).

$$
\begin{aligned}
& v_{1}=\delta \varphi_{1} \quad=\frac{\sin \varphi_{2}}{\sin \Phi} R_{1}^{c}+\frac{\cos \varphi_{2}}{\sin \Phi} R_{2}^{c} \\
& v_{2}=\delta \Phi \quad=\cos \varphi_{2} R_{1}^{c}-\sin \varphi_{2} R_{2}^{c} \\
& v_{3}=\delta \varphi_{2}=-\frac{\sin \varphi_{2}}{\tan \Phi} R_{1}^{c}-\frac{\cos \varphi_{2}}{\tan \Phi} R_{2}^{c}+R_{3}^{c}
\end{aligned}
$$

From eq. 19 it is seen that an exchange of the glide directions $\vec{d}^{n}$ and the glide plane normals $\vec{p}^{n}$ leads to the transposed matrices $E_{j i}^{n}(g)$ in eq. 20 and thus to the same symmetric parts $E_{i j}^{n s}(g)$ in eq. $17 \mathrm{a}$. The solutions of eq. $17 \mathrm{a}, 24$, i.e. the $\gamma_{m}^{n}$ are thus the same. The antisymmetric parts $E_{i j}^{n a}(g)$ in eq. 25 , however, change their signs. If we consider symmetric macroscopic deformation tensors such as the one in eq. 35, then also the rotations $R_{i j}^{m}(g)$ change their signs. In these cases an orientation flow field calculated for the glide systems $\{h k l\}\langle u v w\rangle$ 
describes also the orientation changes for the glide systems $\{u v w\}\langle h k l\rangle$ such that

$$
\vec{v}(g)_{\{u v w\}\langle h k l\rangle}=-\vec{v}(g)_{\{h k l\}\langle u v w\rangle}
$$

\section{Symmetries of the flow field}

The crystals may have a certain crystal symmetry described by the point symmetry group $G^{c}$ which contains symmetry rotations $g^{c}$ and symmetry operations of second kind i.e. inversion axes. A crystal orientation $g$ is thus indistinguishable from the symmetrically equivalent orientations

$$
g^{\prime}=g^{c} \cdot g
$$

where the product means that the two rotations $g$ and $g^{c}$ are carried out one after the other in this sequence.

The macroscopic deformation tensor $\bar{E}_{i j}$ may also have sample symmetry. If we consider, for example, a macroscopic deformation described by

$\bar{E}_{i j}=\left[\begin{array}{rrc}1 & 0 & 0 \\ 0 & -q & 0 \\ 0 & 0 & -(1-q)\end{array}\right]$

then this symmetry is the orthorhombic symmetry containing three two-fold rotation axes in the directions $X_{1}, X_{2}, X_{3}$ respectively.

The symmetry of the deformation tensor may, in general, be described by a symmetry group $G^{s}$ containing the symmetry rotations $g^{s}$ (and possibly symmetry elements of second kind). The crystal orientations

$$
g^{\prime}=g \cdot g^{s}
$$

are then symmetrically equivalent with respect to the symmetry of the deformation tensor.

The rotation vector $\vec{R}$ as a function of the orientation $g$ must obey the crystal as well as the sample symmetry.

If we compare the crystal orientations $g$ and $g^{c} \cdot g$ according to eq. 34 then these are physically indistinguishable orientations and the rotation vector $\vec{R}$ must be the same in both cases

$$
\vec{R}\left(g^{c} \cdot g\right)=\vec{R}(g) \quad \text { crystal symmetry }
$$

If we express the vector by its components in the sample coordinate 
system then this is the same coordinate system in both cases. If, however, it is to be expressed in the crystal coordinate system then the same vector is to be represented in a rotated coordinate system. Hence, eq. 37 can be expressed in components in the sample and the crystal coordinate system.

$$
\begin{gathered}
R_{i}^{s}\left(g^{c} \cdot g\right)=R_{i}^{s}(g) \\
R_{i}^{c}\left(g^{c} \cdot g\right)=o_{j i}\left(g^{c}\right) \cdot R_{j}^{c}(g)
\end{gathered}
$$

Eq. 37 is illustrated in Fig. 6 a.

Similarly, if we consider the rotation vector for the two distinguished orientations $g$ and $g \cdot g^{s}$ related to each other by a symmetry rotation of the deformation symmetry then the rotation vector has also to be rotated through $g^{s}$. Hence, we have in the case of the sample symmetry

$$
\vec{R}\left(g \cdot g^{s}\right)=g^{s} \cdot \vec{R}(g) \quad \text { sample symmetry }
$$

Hence, eq. 38 reads in components in both systems respectively

$$
\begin{gathered}
R_{i}^{s}\left(g \cdot g^{s}\right)=a_{i j}\left(g^{s}\right) R_{j}^{s}(g) \\
R_{i}^{c}\left(g \cdot g^{s}\right)=R_{i}^{c}(g)
\end{gathered}
$$

Eq. 38 is illustrated in Fig. 6 b.
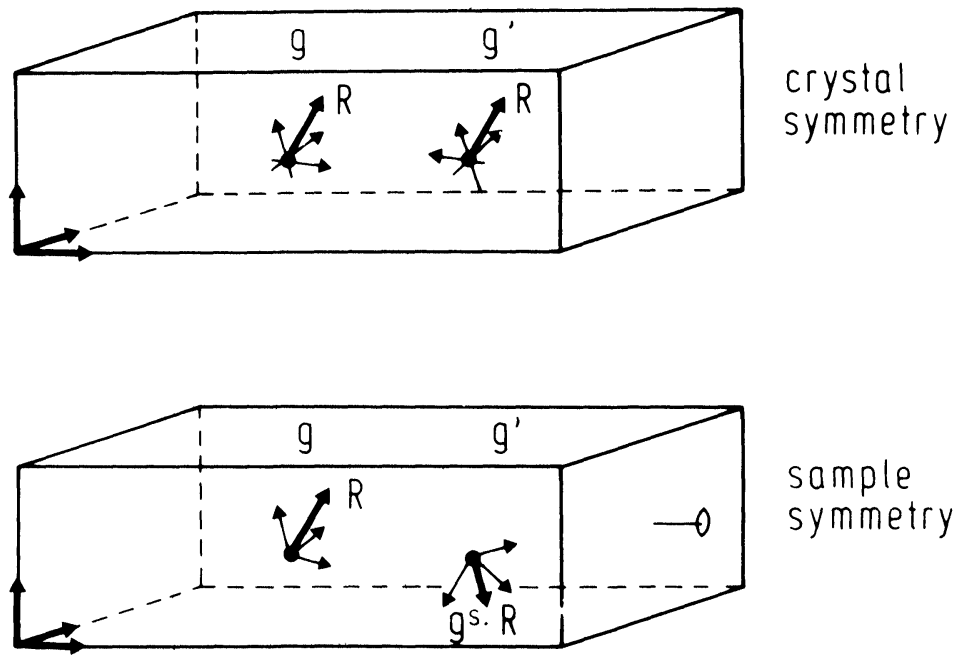

FIGURE 6 The rotation vector $\vec{R}$ in symmetry-related crystal orientations

a) crystal symmetry

b) sample symmetry. 
Hence, the components of the rotation vector in the sample coordinate system, $R_{i}^{s}(g)$ obey the crystal symmetry but not the sample symmetry. On the other hand the components in the crystal coordinate system $R_{i}^{c}(g)$ obey the sample symmetry but not the crystal symmetry (Dahlem 1984, Esling, Bunge 1984).

The components of the rotation vector can be developed into series of generalized spherical harmonics of the corresponding symmetries according to eq. $37 \mathrm{a}$ and $38 \mathrm{~b}$ (Klein 1984)

$$
\begin{aligned}
& R_{i}^{s}(g)=\sum_{l=0}^{\infty} \sum_{\mu=1}^{M(l)} \sum_{n=-l}^{+l} A_{l, i, s}^{\mu \eta} \dot{T}_{l}{ }_{l}^{\mu \eta}(g) \\
& R_{i}^{c}(g)=\sum_{l=0}^{\infty} \sum_{m=-l}^{+l} \sum_{v=1}^{N(l)} A_{l, i, c}^{m v} \dot{T}_{l}{ }^{m v}(g)
\end{aligned}
$$

where $T_{l}^{\mu n}(g)$ are generalized spherical harmonics of the crystal symmetry and $\dot{T}_{l}^{m v}(g)$ those of the sample symmetry.

\section{Numerical calculation of the flow field}

The flow field $v(g)$ was calculated numerically using the Taylor theory described in eq. 16-25,32 with the assumption eq. 28 for $\{111\}\langle 110\rangle$ glide and a deformation tensor eq. 35 with $q=0$ corresponding to plane strain deformation (Bunge 1970). The calculations were carried out in steps of 10 degrees in the Euler angles $\varphi_{1} \Phi \varphi_{2}$ in the range from $0^{\circ}$ to $90^{\circ}$. The components of the rotation vector were then developed into series according to eq. $39 \mathrm{a}$ in order to interpolate them in $5^{\circ}$-steps. The results are shown in Fig. 7 in $\varphi_{1}, \Phi$ and $\varphi_{2}$ sections respectively. Each section contains the components of the flow vector parallel to this section. The absolute value of the vector $v(g)$ is represented in Fig. 8 in equi-level lines. 


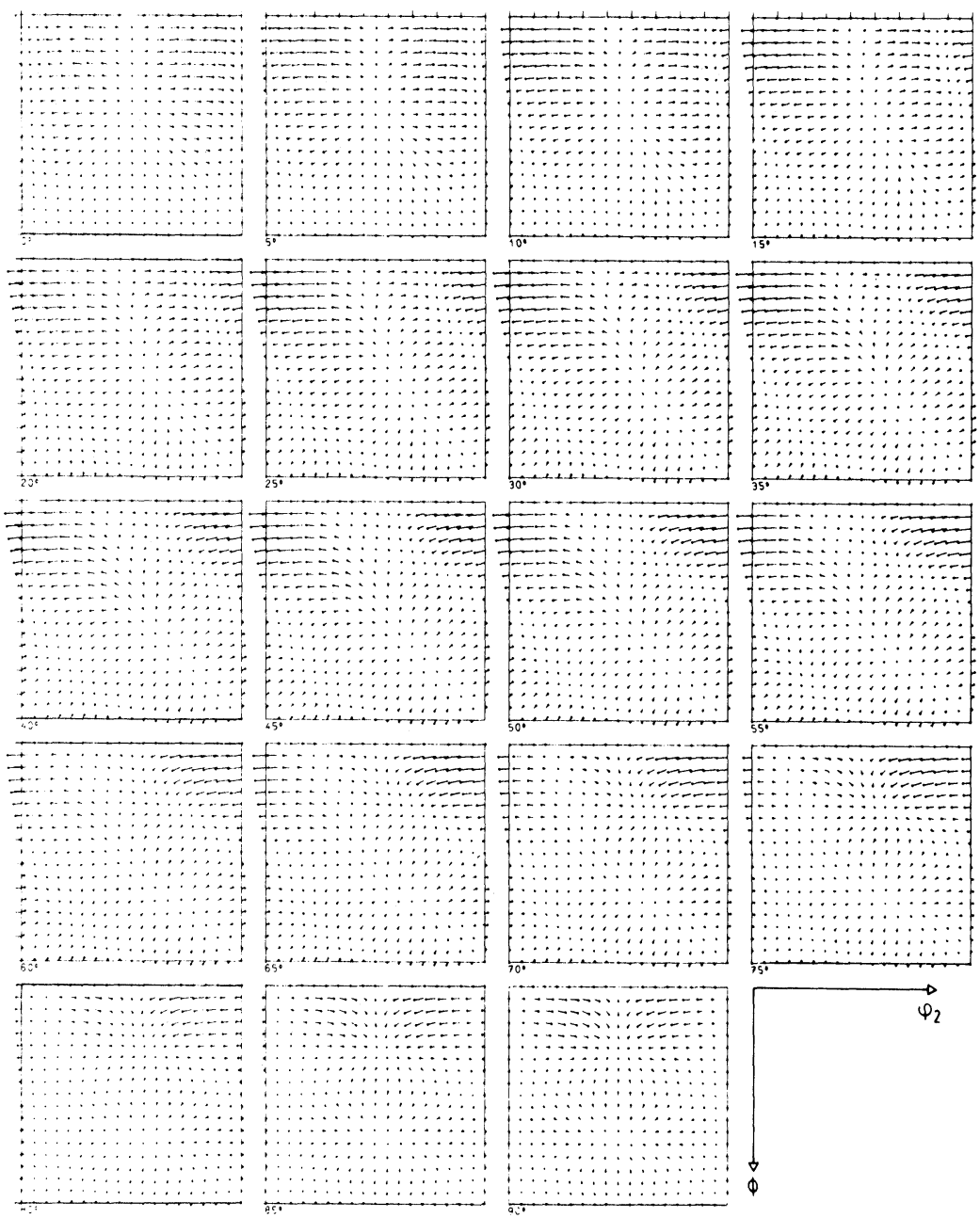

FIGURE 7 The flow field $v(g)$ in the orientation space according to the Taylor theory with $\{111\}\langle 110\rangle$-glide and plane strain deformation $q=0$. In each section the components parallel to the section are represented. Interpolation of the original $10^{\circ}$ calculation by the series expansion eq. 39a.
a) $\varphi_{1}$-sections
b) $\Phi$-sections
c) $\varphi_{2}$-sections 
ORIENTATION FLOW FIELD
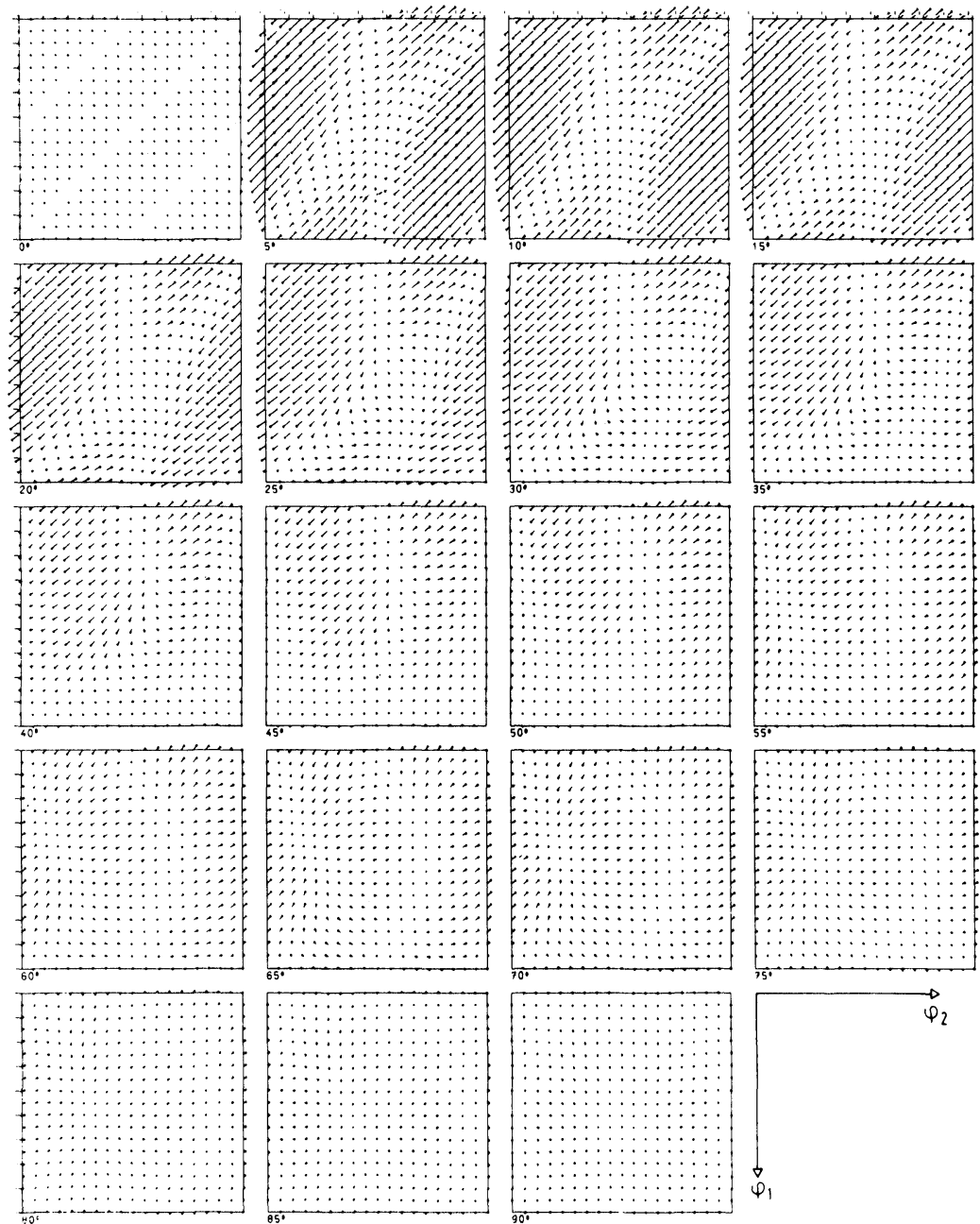
H. J. BUNGE et al.
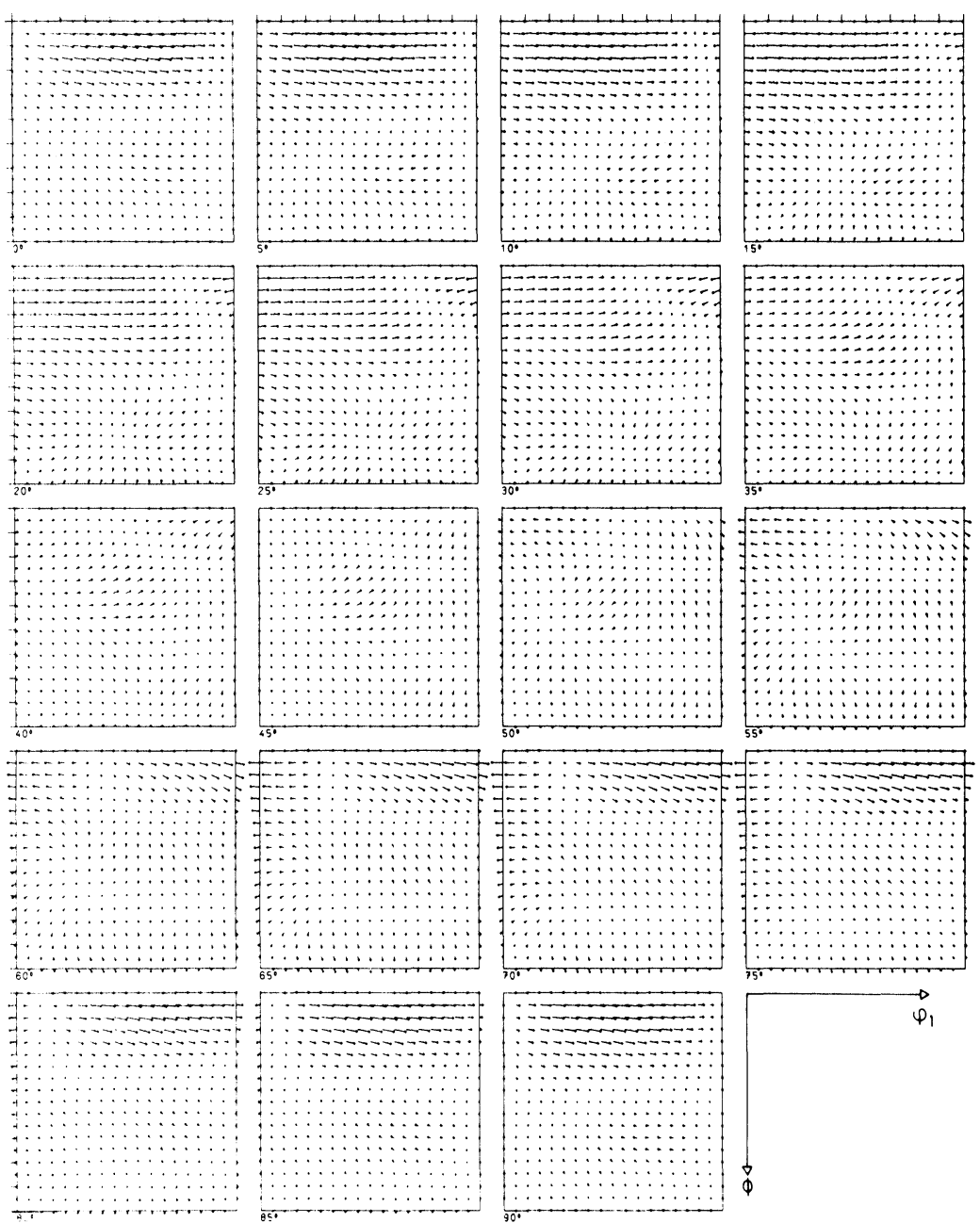

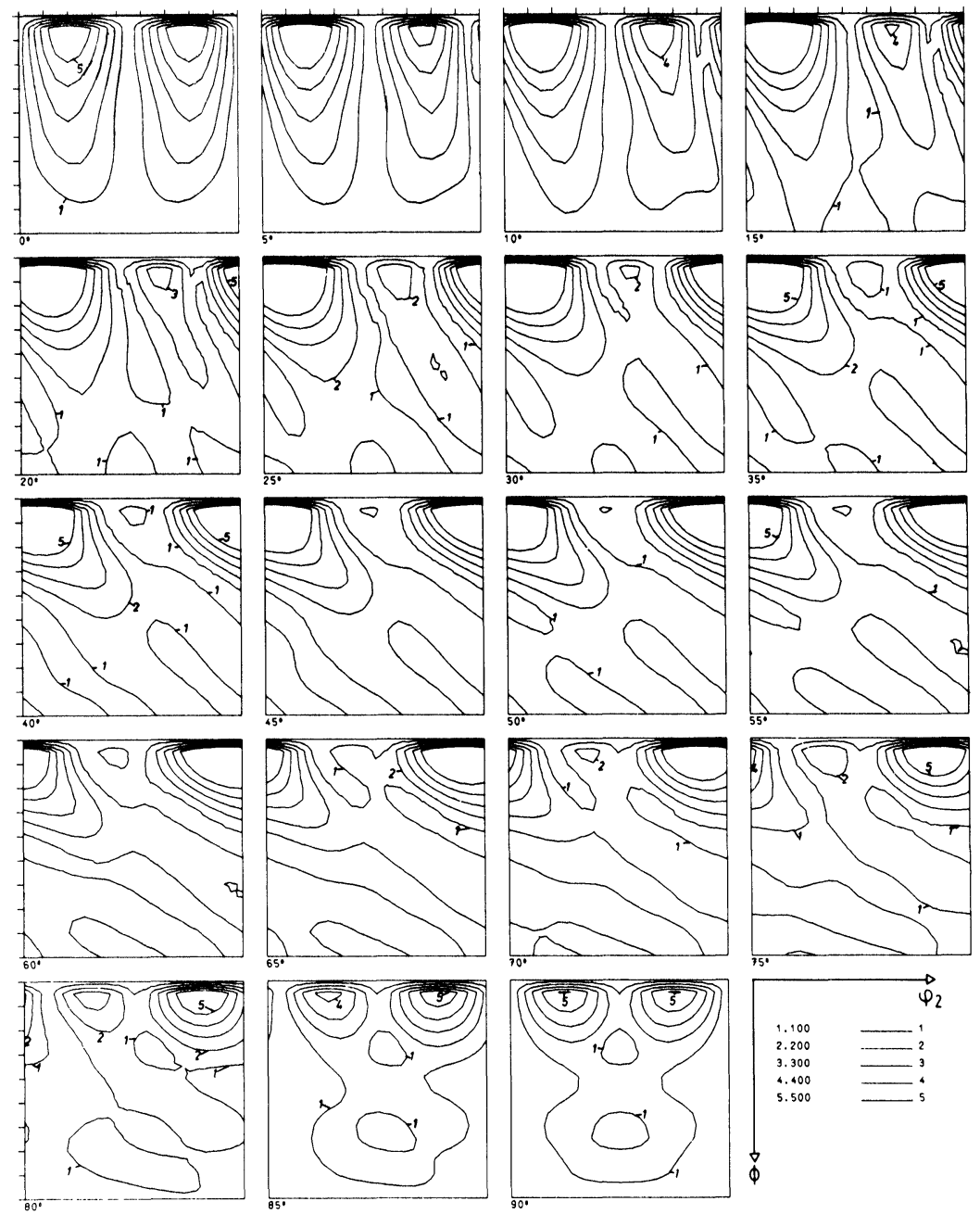

FIGURE 8 The absolute value of the flow vector $v(g)$, Fig. 7 in the three sections

a) $\varphi_{1}$-section

b) $\Phi$-section

c) $\varphi_{2}$-section. 
198
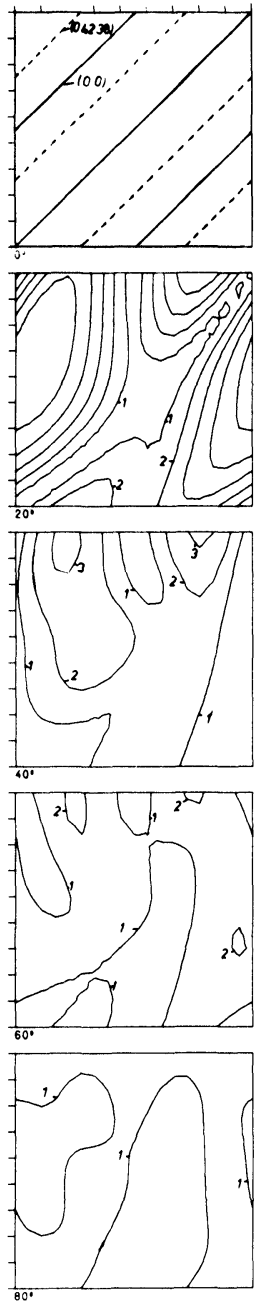

H. J. BUNGE et al.
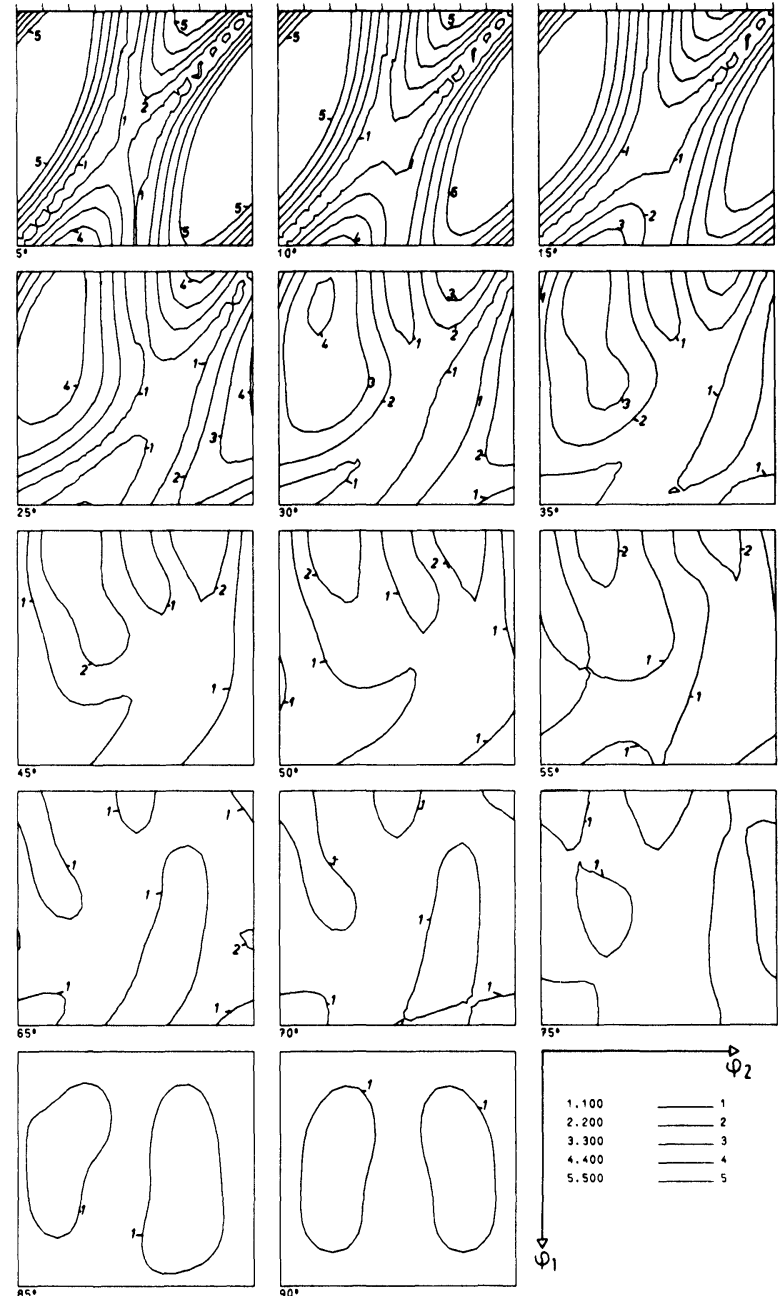
ORIENTATION FLOW FIELD
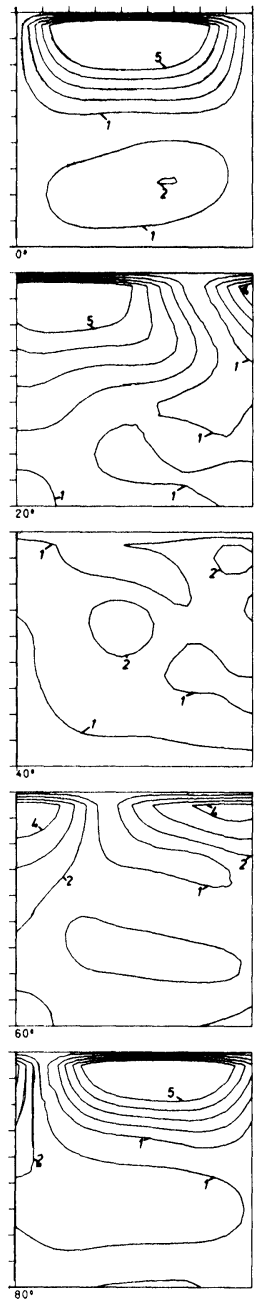
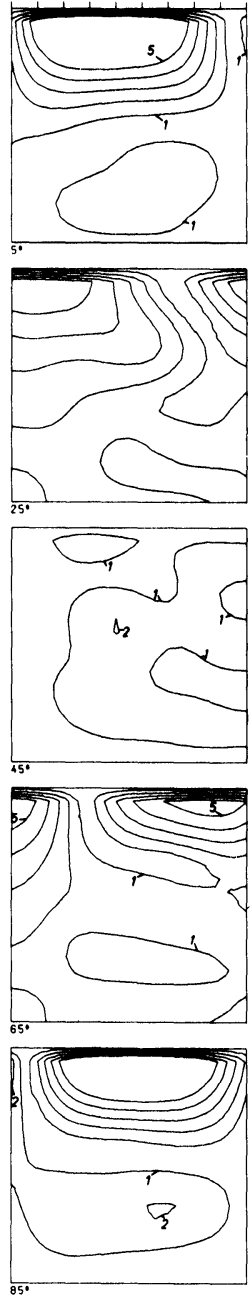
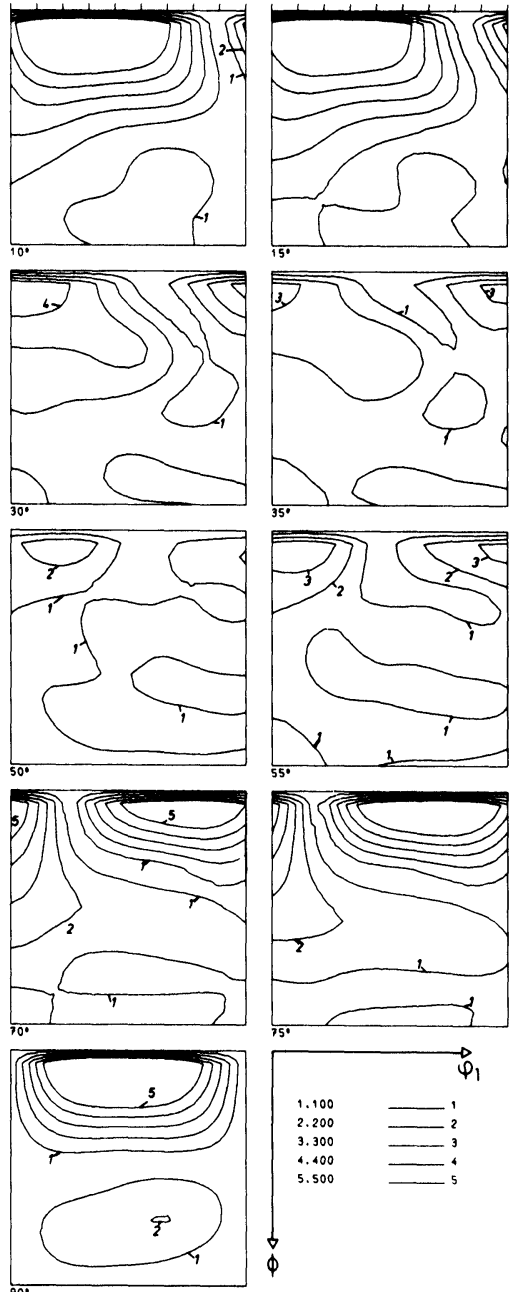


\section{References}

Berveiller, M. and Zaoui, A. in: Textures of Materials, Vol I, p. 319-327, Ed. G. Gottstein and K. Lücke, Spinger Verlag, Berlin (1978).

Bunge, H. J. Kristall und Techn. 5, 145-175 (1970).

Bunge, H. J. Texture Analysis in Material Science-Mathematical Methods, Butterworths, London (1982).

Bunge, H. J. and Esling, C. Scripta Met. 18, 191-195 (1984).

Chin, G. Y. in: Textures in Research and Practice, p. 51-80, Ed. J. Grewen and G. Wassermann, Springer Verlag, Berlin (1969).

Clement, A. Thesis, Toulouse (1980).

Clement, A. and Coulomb, P. in: Textures of Materials, Vol I, p. 329-335, Ed. G. Gottstein and K. Lücke, Springer Verlag, Berlin (1978).

Clement, A. and Coulomb, P. Scipta Met. 13, 899-901 (1979).

Dahlem, E. Diploma Thesis Clausthal (1984).

Esling, C., Bunge, H. J. and Muller, J. in: Proc. 7th Intern. Conf. on Textures of Materials, p. 71-76. Nordwijkerhout (1984).

Gil Sevillano, J., van Houtte, P. and Aernoudt, E. Large Strain Work Hardening and Textures. Progress in Materials Science 25 (1980).

Klein, H. Diploma Thesis Clausthal (1984).

Kocks, U. F. Met. Trans., 1, 1121-1143 (1970).

Mecking, H. Proc. 6th Intern. Conf. on Textures of Materials, p. 53-64. Tokyo (1981).

Taylor, G. I. J. Inst. Metals 62, 307-324 (1938).

Van Houtte, P. Proc. 7th. Intern. Conf. on Textures of Materials p. 7-23, Nordwijkerhout (1984). 